\title{
On a subclass of meromorphically starlike functions with two fixed positive coefficients
}

\author{
Danyal Soybaş ${ }^{\mathrm{a}, *}$, Santosh Joshi ${ }^{\mathrm{b}}$, Haridas Pawar \\ ${ }^{a}$ Department of Mathematics Education, Faculty of Education, Erciyes University, Kayseri 38039, Turkey. \\ ${ }^{b}$ Department of Mathematics, Walchand College of Engineering, Sangli 416415, India. \\ 'SVERI's College of Engineering Pandharpur, Pandharpur 413304, India.
}

\begin{abstract}
In this paper we obtain coefficient inequalities for the subclass of meromorphic univalent functions with two fixed positive coefficients defined in punctured unit disc. We have studied basic properties such as coefficient estimates, radius of convexity, and closure theorems.
\end{abstract}

Keywords: Univalent function, fixed coefficients, distortion theorem.

2010 MSC: 30C45.

\section{Introduction}

Let $\sum$ denote the class of functions of the form

$$
f(z)=\frac{1}{z}+\sum_{n=1}^{\infty} a_{n} z^{n}
$$

which are regular in $\mathrm{U}=\{z: 0<|z|<1\}$ having simple pole at $\mathrm{z}=0$ and residue 1 . Also let $\sum_{\mathrm{p}}$ denote the class of functions of the form

$$
f(z)=\frac{1}{z}+\sum_{n=1}^{\infty}\left|a_{n}\right| z^{n},
$$

which are analytic and univalent in $\mathrm{U}$.

A function $f \in \Sigma_{p}$ satisfying the condition

$$
\operatorname{Re}\left(\frac{z f^{\prime}(z)}{f(z)}\right)<-\alpha,|z|<1
$$

\footnotetext{
*Corresponding author

Email addresses: danyal@erciyes.edu.tr (Danyal Soybaş), joshisb@hotmail.com (Santosh Joshi),

haridas_pawar007@yahoo.co.in (Haridas Pawar)

doi: $10.22436 /$ jmcs.018.02.11
}

Received 2017-01-02 
is said to be meromorphically starlike of order $\alpha(0 \leqslant \alpha<1)$. Also a function $f \in \Sigma_{p}$ satisfying the condition

$$
\operatorname{Re}\left(1+\frac{z f^{\prime \prime}(z)}{f^{\prime}(z)}\right)<-\alpha,|z|<1
$$

is said to be meromorphically convex of order $\alpha(0 \leqslant \alpha<1)$. Let these classes be denoted respectively by $\sum_{p}^{*}(\alpha)$ and $\sum_{p}^{k}(\alpha)$.

Similar other classes of meromorphically univalent functions have been extensively studied by Aouf and Joshi [1], Silverman and Silvia [3], Uralegaddi [4], and Magesh et al. [2].

With this definitions of $\sum$ and $\sum_{p}$, Aouf and Joshi [1] defined the subclass $\sum(\alpha, \beta, \gamma)$ and $\sum_{p}(\alpha, \beta, \gamma)$ as below.

Definition 1.1 ([1]). A function $\mathrm{f}(z) \in \sum$ is said to be in the class $\sum(\alpha, \beta, \gamma)$ if it satisfies the condition

$$
\left|\frac{\frac{z f^{\prime}(z)}{f(z)}+1}{2 \gamma\left[\frac{z f^{\prime}(z)}{f(z)}+\alpha\right]-\left[\frac{z f^{\prime}(z)}{f(z)}+1\right]}\right|<\beta, \quad z \in \mathrm{U},
$$

where $0 \leqslant \alpha<1,0<\beta \leqslant 1$ and $\frac{1}{2}<\gamma \leqslant 1$ if $\alpha=0, \frac{1}{2}<\gamma \leqslant \frac{1}{2 \alpha}$ if $\alpha \neq 0$.

Aouf and Joshi [1] proved the following coefficient estimates for the class $\sum(\alpha, \beta, \gamma)$.

Theorem 1.2 ([1]). Let the function $\mathrm{f}(z)$ defined by (1.1) be analytic in $\mathrm{U}$. If

$$
\sum_{n=1}^{\infty}\{(n+1)+\beta[(1-n)+2 \gamma(n-\alpha)]\}\left|a_{n}\right| \leqslant 2 \beta \gamma(1-\alpha)
$$

with the same constraints as in above definition, then $f \in \sum(\alpha, \beta, \gamma)$.

Theorem 1.3 ([1]). Let the function $\mathrm{f}(z)$ defined by (1.2) be analytic in $\mathrm{U}$. Then $\mathrm{f}(z) \in \sum(\alpha, \beta, \gamma)$ if and only if it satisfies

$$
\sum_{n=1}^{\infty}\{(n+1)+\beta[(1-n)+2 \gamma(n-\alpha)]\}\left|a_{n}\right| \leqslant 2 \beta \gamma(1-\alpha)
$$

with the same constraints as in above definition.

Also, Aouf and Joshi [1] defined the subclass $\sum_{p}(\alpha, \beta, \gamma)$ of $\sum(\alpha, \beta, \gamma)$ for the functions of the form (1.2) in $\sum(\alpha, \beta, \gamma)$ as below.

Definition 1.4 ([1]). Let $\sum_{p}(\alpha, \beta, \gamma)$ be the subclass of $\sum(\alpha, \beta, \gamma)$ consisting of functions of the form

$$
f(z)=\frac{1}{z}+\frac{p \beta \gamma(1-\alpha)}{1+\beta \gamma(1-\alpha)} z+\sum_{n=2}^{\infty}\left|a_{n}\right| z^{n},
$$

where $0 \leqslant p \leqslant 1$.

The coefficient inequality for the class $\Sigma_{p}(\alpha, \beta, \gamma)$ is as below.

Theorem 1.5 ([1]). Let the function $f(z)$ defined by (1.3). Then $f(z) \in \Sigma_{p}(\alpha, \beta, \gamma)$ if and only if

$$
\sum_{n=2}^{\infty}\{(n+1)+\beta[(1-n)+2 \gamma(n-\alpha)]\}\left|a_{n}\right| \leqslant 2 \beta \gamma(1-\alpha)(1-p) .
$$


Aouf and Joshi [1] also remarked that the function $f(z)$ defined by (1.3) is in the class $f(z) \in \sum_{p}(\alpha, \beta, \gamma)$, then

$$
\left|a_{n}\right| \leqslant \frac{2 \beta \gamma(1-\alpha)(1-p)}{\{(n+1)+\beta[(1-n)+2 \gamma(n-\alpha)]\}}, \quad n=2,3, \ldots
$$

In the view of above inequality, we can see that $f(z)$ defined by (1.3) is in the class $\Sigma_{p}(\alpha, \beta, \gamma)$ satisfying

$$
\left|a_{2}\right| \leqslant \frac{2 \beta \gamma(1-\alpha)(1-p)}{3+\beta[2 \gamma(2-\alpha)-1]} .
$$

Hence we may take

$$
\left|a_{2}\right|=\frac{2 q \beta \gamma(1-\alpha)(1-p)}{3+\beta[2 \gamma(2-\alpha)-1]}
$$

where $0 \leqslant q \leqslant 1$.

By virtue of above equality, we introduce the following class of analytic functions with two fixed coefficients.

Definition 1.6. Let $\sum_{p}^{q}(\alpha, \beta, \gamma)$ be the subclass of $\sum_{p}(\alpha, \beta, \gamma)$ consisting of functions of the form

$$
f(z)=\frac{1}{z}+\frac{p \beta \gamma(1-\alpha)}{1+\beta \gamma(1-\alpha)} z+\frac{2 q \beta \gamma(1-\alpha)(1-p)}{3+\beta[2 \gamma(2-\alpha)-1]} z^{2}+\sum_{n=3}^{\infty}\left|a_{n}\right| z^{n} .
$$

\section{Results and discussions}

2.1. Sharpness theorems

Theorem 2.1. Let the function $f(z)$ defined by (1.4). Then $f(z) \in \sum_{p}^{q}(\alpha, \beta, \gamma)$ if and only if

$$
\sum_{n=3}^{\infty}\{(n+1)+\beta[(1-n)+2 \gamma(n-\alpha)]\}\left|a_{n}\right| \leqslant 2 \beta \gamma(1-\alpha)(1-p)(1-q) .
$$

The result (2.1) is sharp.

Proof. Let

$$
f(z)=\frac{1}{z}+\frac{p \beta \gamma(1-\alpha)}{1+\beta \gamma(1-\alpha)} z+\frac{2 q \beta \gamma(1-\alpha)(1-p)}{3+\beta[2 \gamma(2-\alpha)-1]} z^{2}+\sum_{n=3}^{\infty}\left|a_{n}\right| z^{n}
$$

belong to the class $\sum_{p}^{q}(\alpha, \beta, \gamma)$. Putting $a_{2}=\frac{2 q \beta \gamma(1-\alpha)(1-p)}{3+\beta[2 \gamma(2-\alpha)-1]}$ in Theorem 1.5, we have

$$
\begin{aligned}
\{3+\beta[2 \gamma(2-\alpha)-1]\} \frac{2 q \beta \gamma(1-\alpha)(1-p)}{3+\beta[2 \gamma(2-\alpha)-1]}+ & \sum_{n=3}^{\infty}\{(n+1)+\beta[(1-n)+2 \gamma(n-\alpha)]\}\left|a_{n}\right| \\
& \leqslant 2 \beta \gamma(1-\alpha)(1-p), \\
\sum_{n=3}^{\infty}\{(n+1)+\beta[(1-n)+2 \gamma(n-\alpha)]\}\left|a_{n}\right| & \leqslant 2 \beta \gamma(1-\alpha)(1-p)-2 q \beta \gamma(1-\alpha)(1-p), \\
\sum_{n=3}^{\infty}\{(n+1)+\beta[(1-n)+2 \gamma(n-\alpha)]\}\left|a_{n}\right| & \leqslant 2 \beta \gamma(1-\alpha)(1-p)(1-q) .
\end{aligned}
$$

Hence the first part of the proof is completed. The result is sharp for the function

$$
f(z)=\frac{1}{z}+\frac{p \beta \gamma(1-\alpha)}{1+\beta \gamma(1-\alpha)} z+\frac{2 q \beta \gamma(1-\alpha)(1-p)}{3+\beta[2 \gamma(2-\alpha)-1]} z^{2}+\frac{2 \beta \gamma(1-\alpha)(1-p)(1-q)}{(n+1)+\beta[(1-n)+2 \gamma(n-\alpha)]} z^{n}
$$

for $n \geqslant 3$. 
In the next Theorem, we find the radius of convexity for a function $f(z)$ to be in the class $\sum_{p}^{q}(\alpha, \beta, \gamma)$.

Theorem 2.2. If the function $\mathrm{f}(z)$ defined by (1.4) is in the class $\sum_{\mathrm{p}}^{\mathrm{q}}(\alpha, \beta, \gamma)$, then $\mathrm{f}(z)$ is convex in $0<|z|<$ $r(\alpha, \beta, \gamma, p, q)$, where $r(\alpha, \beta, \gamma, p, q)$ is the largest value for which

$$
\frac{3 p \beta \gamma(1-\alpha)}{1+\beta \gamma(1-\alpha)} r^{2}+\frac{12 q \beta \gamma(1-\alpha)(1-p)}{3+\beta[2 \gamma(2-\alpha)-1]} r^{3}+\frac{2 n(n+2) \beta \gamma(1-\alpha)(1-p)(1-q)}{(n+1)+\beta[(1-n)+2 \gamma(n-\alpha)]} r^{n+1} \leqslant 1,
$$

where $\mathrm{n}=3,4, \ldots$.

The result is sharp for the function $\mathrm{f}(z)$ given by (2.2).

Proof. To find out radius of convexity we make use of same techniques used by Uralegaddi [4].

It is enough to show that

$$
\left|\frac{z f^{\prime \prime}(z)}{f^{\prime}(z)}+2\right|<1
$$

The function

$$
f(z)=\frac{1}{z}+\frac{p \beta \gamma(1-\alpha)}{1+\beta \gamma(1-\alpha)} z+\frac{2 q \beta \gamma(1-\alpha)(1-p)}{3+\beta[2 \gamma(2-\alpha)-1]} z^{2}+\sum_{n=3}^{\infty}\left|a_{n}\right| z^{n}
$$

will be convex in any annulus $0<|z|<r$ for which $\left|\frac{z f^{\prime \prime}(z)}{f^{\prime}(z)}+2\right|<1$. But

$$
\left|\frac{z f^{\prime \prime}(z)}{f^{\prime}(z)}+2\right|=\left|\frac{\frac{2 p \beta \gamma(1-\alpha)}{1+\beta \gamma(1-\alpha)} r^{2}+\frac{8 q \beta \gamma(1-\alpha)(1-p)}{3+\beta[2 \gamma(2-\alpha)-1]} r^{3}+\sum_{n=3}^{\infty} n(n+1)\left|a_{n}\right| r^{n+1}}{1-\frac{p \beta \gamma(1-\alpha)}{1+\beta \gamma(1-\alpha)} r^{2}-\frac{4 q \beta \gamma(1-\alpha)(1-p)}{3+\beta[2 \gamma(2-\alpha)-1]} r^{3}-\sum_{n=3}^{\infty} n\left|a_{n}\right| r^{n+1}}\right|<1
$$

Above inequality holds true if

$$
\frac{3 p \beta \gamma(1-\alpha)}{1+\beta \gamma(1-\alpha)} r^{2}+\frac{12 q \beta \gamma(1-\alpha)(1-p)}{3+\beta[2 \gamma(2-\alpha)-1]} r^{3}+\sum_{n=3}^{\infty} n(n+2)\left|a_{n}\right| r^{n+1}<1
$$

Since the function $f(z) \in \sum_{p}^{q}(\alpha, \beta, \gamma)$, from Theorem 2.1 we may take

$$
\sum_{n=3}^{\infty}\left|a_{n}\right|=\frac{2 \beta \gamma(1-\alpha)(1-p)(1-q)}{\{(n+1)+\beta[(1-n)+2 \gamma(n-\alpha)]\}} \lambda_{n}, \quad n \geqslant 3
$$

where $\lambda_{n} \geqslant 0$ and $\sum_{n=3}^{\infty} \lambda_{n} \leqslant 1$. For each fixed $r$, choose an integer $n=n(r)$ for which $\frac{n(n+2) r^{n+1}}{\{(n+1)+\beta[(1-n)+2 \gamma(n-\alpha)]\}}$ is maximal. Then it follows that

$$
\sum_{n=3}^{\infty} n(n+2)\left|a_{n}\right| r^{n+1} \leqslant \frac{2 n(n+2) \beta \gamma(1-\alpha)(1-p)(1-q)}{\{(n+1)+\beta[(1-n)+2 \gamma(n-\alpha)]\}} r^{n+1} .
$$

Hence $f(z)$ is convex in $0<|z|<r(\alpha, \beta, \gamma, p, q)$ provided that

$$
\frac{3 p \beta \gamma(1-\alpha)}{1+\beta \gamma(1-\alpha)} r^{2}+\frac{12 q \beta \gamma(1-\alpha)(1-p)}{3+\beta[2 \gamma(2-\alpha)-1]} r^{3}+\frac{2 n(n+2) \beta \gamma(1-\alpha)(1-p)(1-q)}{\{(n+1)+\beta[(1-n)+2 \gamma(n-\alpha)]\}} r^{n+1} \leqslant 1 .
$$

Now find the value of $r_{0}=r_{0}(\alpha, \beta, \gamma, p, q)$ and corresponding $n\left(r_{0}\right)$ so that

$$
\frac{3 p \beta \gamma(1-\alpha)}{1+\beta \gamma(1-\alpha)} r_{0}^{2}+\frac{12 q \beta \gamma(1-\alpha)(1-p)}{3+\beta[2 \gamma(2-\alpha)-1]} r_{0}^{3}+\frac{2 n(n+2) \beta \gamma(1-\alpha)(1-p)(1-q)}{\{(n+1)+\beta[(1-n)+2 \gamma(n-\alpha)]\}} r_{0}^{n+1}=1 .
$$

It is the value of $r_{0}$ for which the function $f(z)$ is convex in $0<|z|<r_{0}$. 


\subsection{Closure theorems}

In this section, we shall prove that the class $\sum_{\mathfrak{p}}^{q}(\alpha, \beta, \gamma)$ is closed under linear combinations.

Theorem 2.3. The class $\sum_{\mathfrak{p}}^{\mathrm{q}}(\alpha, \beta, \gamma)$ is closed under linear combinations.

Proof. Suppose $\sum_{p}^{q}(\alpha, \beta, \gamma)$, then

$$
f(z)=\frac{1}{z}+\frac{p \beta \gamma(1-\alpha)}{1+\beta \gamma(1-\alpha)} z+\frac{2 q \beta \gamma(1-\alpha)(1-p)}{3+\beta[2 \gamma(2-\alpha)-1]} z^{2}+\sum_{n=3}^{\infty}\left|a_{n}\right| z^{n}
$$

and

$$
g(z)=\frac{1}{z}+\frac{p \beta \gamma(1-\alpha)}{1+\beta \gamma(1-\alpha)} z+\frac{2 q \beta \gamma(1-\alpha)(1-p)}{3+\beta[2 \gamma(2-\alpha)-1]} z^{2}+\sum_{n=3}^{\infty}\left|b_{n}\right| z^{n} .
$$

It is sufficient to prove that the function $\mathrm{H}$ defined by

$$
H(z)=\lambda f(z)+(1-\lambda) g(z), \quad(0 \leqslant \lambda \leqslant 1)
$$

is also in the class $\sum_{\mathrm{p}}^{\mathrm{q}}(\alpha, \beta, \gamma)$. Since

$$
\mathrm{H}(z)=\frac{1}{z}+\frac{p \beta \gamma(1-\alpha)}{1+\beta \gamma(1-\alpha)} z+\frac{2 q \beta \gamma(1-\alpha)(1-p)}{3+\beta[2 \gamma(2-\alpha)-1]} z^{2}+\sum_{k=3}^{\infty}\left[\left|a_{n}\right|+(1-\lambda)\left|b_{n}\right|\right] z^{n},
$$

we observe that

$$
\sum_{n=3}^{\infty}\{(n+1)+\beta[(1-n)+2 \gamma(n-\alpha)]\}\left[\left|a_{n}\right|+(1-\lambda)\left|b_{n}\right|\right] \leqslant 2 \beta \gamma(1-\alpha)(1-p)(1-q) .
$$

Thus $H \in \sum_{p}^{q}(\alpha, \beta, \gamma)$, which completes the proof.

Theorem 2.4. If

$$
f_{2}(z)=\frac{1}{z}+\frac{p \beta \gamma(1-\alpha)}{1+\beta \gamma(1-\alpha)} z+\frac{2 q \beta \gamma(1-\alpha)(1-p)}{3+\beta[2 \gamma(2-\alpha)-1]} z^{2}
$$

and

$$
f_{n}(z)=\frac{1}{z}+\frac{p \beta \gamma(1-\alpha)}{1+\beta \gamma(1-\alpha)} z+\frac{2 q \beta \gamma(1-\alpha)(1-p)}{3+\beta[2 \gamma(2-\alpha)-1]} z^{2}+\frac{2 \beta \gamma(1-\alpha)(1-p)(1-q)}{\{(n+1)+\beta[(1-n)+2 \gamma(n-\alpha)]\}} z^{n},
$$

then $f \in \sum_{\mathrm{p}}^{\mathrm{q}}(\alpha, \beta, \gamma)$ if and only if it can be expressed in the form

$$
f(z)=\sum_{n=2}^{\infty} \lambda_{n} f_{n}(z)
$$

where $\lambda_{n} \geqslant 0$ and $\sum_{n=2}^{\infty} \lambda_{n}=1$.

Proof. Let

$$
\begin{aligned}
& f(z)=\sum_{n=2}^{\infty} \lambda_{n} f_{n}(z), \\
& f(z)=\frac{1}{z}+\frac{p \beta \gamma(1-\alpha)}{1+\beta \gamma(1-\alpha)} z+\frac{2 q \beta \gamma(1-\alpha)(1-p)}{3+\beta[2 \gamma(2-\alpha)-1]} z^{2}+\sum_{n=3}^{\infty} \frac{2 \beta \gamma(1-\alpha)(1-p)(1-q)}{\{(n+1)+\beta[(1-n)+2 \gamma(n-\alpha)]\}} \lambda_{n} z^{n},
\end{aligned}
$$




$$
f(z)=\frac{1}{z}+\frac{p \beta \gamma(1-\alpha)}{1+\beta \gamma(1-\alpha)} z+\frac{2 q \beta \gamma(1-\alpha)(1-p)}{3+\beta[2 \gamma(2-\alpha)-1]} z^{2}+\sum_{n=3}^{\infty} a_{n} z^{n},
$$

where $a_{n}=\sum_{n=3}^{\infty} \frac{2 \beta \gamma(1-\alpha)(1-p)(1-q)}{\{(n+1)+\beta[(1-n)+2 \gamma(n-\alpha)]\}} \lambda_{n} \geqslant 0,(n \geqslant 3)$. Since,

$$
\begin{aligned}
\sum_{n=3}^{\infty} & \{(n+1)+\beta[(1-n)+2 \gamma(n-\alpha)]\}\left|a_{n}\right| \\
& =\sum_{n=3}^{\infty}\{(n+1)+\beta[(1-n)+2 \gamma(n-\alpha)]\} \frac{2 \beta \gamma(1-\alpha)(1-p)(1-q)}{\{(n+1)+\beta[(1-n)+2 \gamma(n-\alpha)]\}} \lambda_{n} \\
& =2 \beta \gamma(1-\alpha)(1-p)(1-q) \sum_{n=3}^{\infty} \lambda_{n} \leqslant 2 \beta \gamma(1-\alpha)(1-p)(1-q),
\end{aligned}
$$

we have $f(z) \in \sum_{p}^{q}(\alpha, \beta, \gamma)$.

Conversely, suppose that $f \in \sum_{p}^{q}(\alpha, \beta, \gamma)$ and since

$$
a_{n} \leqslant \frac{2 \beta \gamma(1-\alpha)(1-p)(1-q)}{\{(n+1)+\beta[(1-n)+2 \gamma(n-\alpha)]\}}, \quad(n \geqslant 3),
$$

setting

$$
\lambda_{n}=\frac{2 \beta \gamma(1-\alpha)(1-p)(1-q)}{\{(n+1)+\beta[(1-n)+2 \gamma(n-\alpha)]\}}\left|a_{n}\right|, \quad(n \geqslant 3),
$$

and

$$
\lambda_{2}=1-\sum_{n=3}^{\infty} \lambda_{n}
$$

it follows that

$$
f(z)=\sum_{n=2}^{\infty} \lambda_{n} f_{n}(z)
$$

Hence proof is completed.

\section{Conclusions}

Classes of meromorphically univalent functions have been studied for a few decades. Obtaining coefficient inequalities for the subclass of meromorphic univalent function with two fixed positive coefficients defined in punctured unit disc, which is an preferential topic concerning inequalities in complex analysis, is investigated to some extent in this work. Because basic properties such as coefficient estimates, radius of convexity, and closure theorems are obtained in this study, it may be considered as a useful tool for those who are interested in above-mentioned topics for further research.

\section{References}

[1] M. K. Aouf, S. B. Joshi, On certain subclasses of meromorphically starlike functions with positive coefficients, Soochow J. Math., 24 (1998), 79-90. 1, 1.1, 1, 1.2, 1.3, 1, 1.4, 1.5, 1

[2] N. Magesh, N. B. Gatti, S. Mayilvaganan, On Certain Subclasses of Meromorphic Functions with Positive and Fixed Second Coefficients Involving the Liu-Srivastava Linear Operator, ISRN Math. Anal., 2012 (2012), 11 pages. 1

[3] H. Silverman, E. M. Silvia, Fixed coefficients for subclasses of starlike functions, Houston J. Math., 7 (1981), 129-136. 1

[4] B. A. Uralegaddi, Meromorphically starlike functions with positive and fixed second coefficients, Kyungpook Math. J., 29 (1989), 64-68. 1, 2.1 